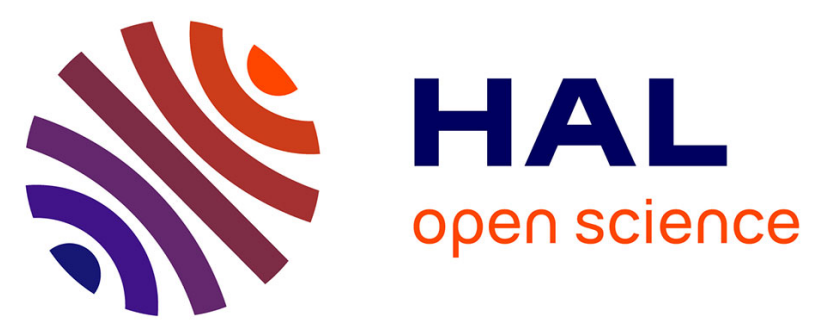

\title{
Synthesis and Characterization of Molecularly Imprinted Polymers for the Selective Extraction of Carbamazepine and Analogs from Human Urine Samples
}

\author{
Audrey Combès, Porkodi Kadhirvel, Louis Bordron, Valérie Pichon
}

\section{- To cite this version:}

Audrey Combès, Porkodi Kadhirvel, Louis Bordron, Valérie Pichon. Synthesis and Characterization of Molecularly Imprinted Polymers for the Selective Extraction of Carbamazepine and Analogs from Human Urine Samples. Chromatographia, 2019, 82 (1), pp.287-295. 10.1007/s10337-018-3680-4 . hal-02093842

\section{HAL Id: hal-02093842 \\ https://hal.sorbonne-universite.fr/hal-02093842}

Submitted on 9 Apr 2019

HAL is a multi-disciplinary open access archive for the deposit and dissemination of scientific research documents, whether they are published or not. The documents may come from teaching and research institutions in France or abroad, or from public or private research centers.
L'archive ouverte pluridisciplinaire HAL, est destinée au dépôt et à la diffusion de documents scientifiques de niveau recherche, publiés ou non, émanant des établissements d'enseignement et de recherche français ou étrangers, des laboratoires publics ou privés. 
Chromatographia (2019) 82:287-295

https://doi.org/10.1007/s10337-018-3680-4

3

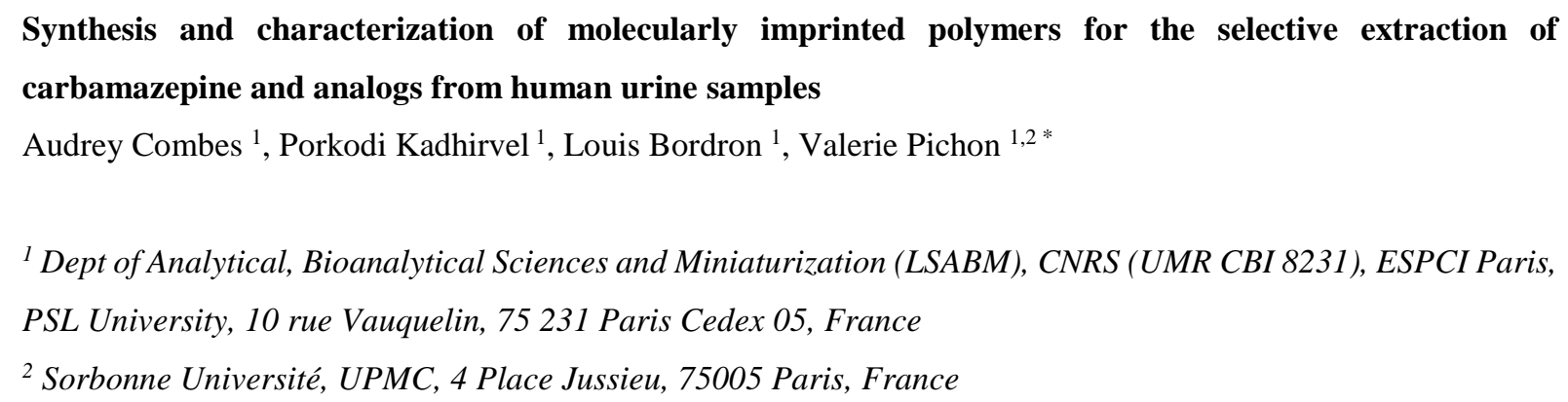

Corresponding author institution: LSABM, ESPCI-Paris, 10 rue Vauquelin, 75005 Paris, France.

\begin{abstract}
Two molecularly imprinted polymers (MIPs) were synthesized according to a previous work from our group dealing with the extraction of carbamazepine from environmental waters. The potential of these MIPs, that differ by the nature of the monomer used for their synthesis, to selectively extract the drugs carbamazepine and oxcarbazepine and the metabolite 10,11-epoxycarbamazepine was first studied in spiked pure water and a high selectivity was obtained with both MIPs for the three target molecules in this pure media. This selectivity was maintained when applying one of these MIPs to urine samples. Indeed, recoveries of extraction were higher than $82 \%$ on the MIP and lower than $20 \%$ on the corresponding non-imprinted polymer used as a control. The repeatability of the extraction procedure applied to urine was also demonstrated with RSD below $20 \%$ for the recoveries of extraction of the three targets at a spiking level of $20 \mathrm{ng} . \mathrm{L}^{-1}$. Limits of quantification between 1 and 7 ng. $\mathrm{L}^{-1}$ were determined for urine sample using the MIP as extraction sorbent combined with LC-MS analysis. The potential of the MIP was compared to the one of Oasis HLB sorbent. This study showed that the MIP constitutes a powerful tool to avoid matrix effects encountered for the quantification of the target molecules in urine sample extracted on Oasis HLB.
\end{abstract}

Keywords: Molecularly imprinted polymers; Carbamazepine; Oxcarbazepine; Metabolite; Urine; LC-MS analysis.

\title{
Introduction
}

Carbamazepine (CBZ) is the most commonly used drug to treat partial epileptic seizures. Oxcarbazepine (OXC) is a structural derivative of carbamazepine, with a ketone on the dibenzazepine ring that was developed with the intention to be as effective as carbamazepine while causing fewer side effects. Because of their high intake, they are frequently detected in the urban waste water cycle due to their excretion in urine and feces [1]. Carbamazepine is metabolized in the liver, the primary metabolic pathway being a conversion to 10,11-epoxycarbamazepine (epoCBZ) that can also be found in water samples. Indeed, their concentration levels in German surface water have recently reached 1.64, 0.44 and $0.08 \mu \mathrm{g} / \mathrm{L}$ for CBZ, OXC and epoCBZ respectively in some locations [1]. 
As urine constitutes a major route of contamination of waters, their determination in this biological fluid constitutes an important task.

Their analysis can be achieved by numerous separation methods such as gas, high performance liquid or thin layer chromatography and electrokinetic methods as recently reviewed [2], yet the liquid chromatography coupled to mass spectrometry (LC-MS) remains an unavoidable method because of its high sensitivity and specificity. However, the low levels of concentration that have to be reached combined with the complexity of urine samples implies the use of a sample pretreatment method to concentrate and purify the samples before their analysis. For this, different approaches were proposed such as dilution, protein precipitation, liquid-liquid extraction (LLE), dispersive LLE, solid-phase extraction (SPE), dispersive SPE [3-6]. To improve the sensitivity of this sample pretreatment step by the removal of interfering compounds, it was recently proposed to combine a first extraction using acetonitrile on a freeze-dried sample of urine with a SPE step on a mixed mode sorbent [3]. The improvement in selectivity can also be obtained using molecularly imprinted polymers (MIPs). These polymers are synthesized in the presence of a template molecule, leading to the formation of a polymer that possess cavities that are complementary in size, shape and chemical function of the template compound and that will determine its selectivity. Indeed, the ability of a MIP to selectively recognize and then retain targeted compounds directly depends on the shape and the nature of the chemical function of the cavities that are both fixed by the conditions of synthesis of the MIPs, i.e. the nature of the template, of the monomers, of the cross-linker and of the porogenic solvent.

MIPs have been already developed for the selective extraction of CBZ [7-18] using systematically CBZ as template molecule except in one recent study from our group [18]. Indeed, as the synthesis process of the MIP requires the introduction of a large amount of template molecule, and regarding the targeted concentrations in samples, this choice was not considered as relevant. Indeed, even after a careful washing of the polymer after its synthesis and a rigorous control of this step, the template molecule can still leak from the MIP during subsequent extraction procedures thus leading to false positives. This can be circumvented by selecting a CBZ analog, methoxycarbamazepine (MCBZ), that can be distinguish from CBZ by their different retention time in LC-MS analysis [18]. In most cases, methacrylic acid (MAA) was used as monomer in association with ethylene glycol dimethacrylate (EGDMA) [12, 13, 15, 16], trimethylolpropane trimethacrylate (TRIM) [10, 11] or with divinylbenzene (DVB) [7-9, 14, 18] as cross-linker and mainly in acetonitrile, toluene, chloroform and dichloromethane or in some mixtures of these solvents as porogen. A recent study also reports the synthesis of an imprinted interpenetrating polymer network based on a mix of styrene and tetramethoxysilane [17]. Some of the CBZ MIPs were applied to the extraction of CBZ from urine [7, 8, 16, 17], serum [12], or plasma [16] and environmental waters $[9,13,14,18]$. All these MIPs were synthesized for the selective extraction of CBZ, but few studies reported some results concerning the recognition of its structural analog OXC in water [13, 18], serum $[12]$ and in urine $[8,12]$ with very variable results in terms of selectivity.

The selectivity of a MIP can be evaluated by comparing the extraction recoveries of a target analyte on a MIP with the extraction recoveries obtained using a non-imprinted polymer (NIP). This latter is synthesized in the same conditions as the MIP but without the introduction of the template, the lack of specific cavities in the NIP generating lower extraction recoveries on this sorbent than on the MIP. For water samples, the selectivity was demonstrated for the selective extraction of CBZ, OXC and even epoCBZ (a CBZ metabolite), using a MIP obtained using MAA and DVB for its synthesis [18], no retention and selectivity was obtained using a MIP 
prepared with 2-Vinylpyridine and EGDMA [13]. For biological fluids, high retention of CBZ in serum sample [12] and in urine [7, 8] were reported, even for OXC [8, 12], but without providing any results derived from NIP to control the selectivity. In return, the selective extraction of CBZ from urine was reported by Asgari et al. with recoveries of $91 \%$ and about $20 \%$ on MIP and NIP respectively [17], however no data being provided related the extraction of OXC and CBZ metabolites.

In return, the two MIPs, previously developed by our group using an analog of CBZ as template, using either TFMAA or MAA as monomer and DVB as cross-linker, have shown a high potential for the selective extraction of CBZ, OXC and epoCBZ from mineral and river water samples. Therefore, the objective of this work was to synthesize MIPs in the same conditions and apply them to the selective extraction of CBZ, OXC and epoCBZ from urine. After checking the selectivity of these newly synthesized MIPs in pure aqueous media, an extraction procedure was optimized for the treatment of urine samples. The potential of one of the MIP was then further evaluated by measuring the extraction recoveries of the three target analytes and by comparing its performances to those of a conventional Oasis HLB polymer.

\section{Material and method}

\section{Materials}

Trifluoromethacrylic acid (TFMAA) 98\% was purchased from Apollo Scientific Ltd (Manchester, UK). Carbamazepine (CBZ) 98\%, oxcarbamazepine (OXC) 98\% were procured from ABCR, Karlsruhe, Germany, methoxycarbamazepine (MCBZ) 95\% from Fluorochem Ltd (Derbyshire, UK), methacrylic acid (MAA) 99\%, divinylbenzene (DVB) 80\% and carbamazepine 10,11-epoxide (epoCBZ) 98\% from Sigma-Aldrich (Saint Quentin Fallavier, France). MAA was distilled under vacuum in order to remove inhibitors. Azo-N,N'-bis-isobutyronitrile (AIBN) 98\% was purchased from Acros Organics (Noisy-le-Grand, France). HPLC-grade acetonitrile (ACN), methanol (MeOH), dichloromethane (DCM), hexane and toluene were supplied by Carlo Erba (Val de Reuil, France). Acetic acid (AA) $99.7 \%$ was purchased from VWR (Fontenay-sous-Bois, France). High purity water was dispensed by a Milli-Q purification system (Millipore, Saint Quentin en Yvelines, France).

\section{Instrumentation and analytical conditions}

The LC-MS/MS analyses were performed using the liquid chromatograph (UltiMate 3000®, Thermo Scientific, Illkirch, France) coupled with a Triple Stage Quadrupole Mass Spectrometer (TSQ Quantum Access MAX, Thermo Scientific, Illkirch, France) equipped with a heated electrospray ionization source (HESI2). The LC/MS acquisitions were controlled by the Thermo Xcalibur Control software version 2.2. The chromatographic separation was performed on Varian $\mathrm{C} 18$ Omnispher column $(150 \times 2.1 \mathrm{~mm}, 5 \mu \mathrm{m})$ maintained at $35^{\circ} \mathrm{C}$. The mobile phase, $\mathrm{MeOH} / \mathrm{ACN} / \mathrm{H}_{2} \mathrm{O}(38 / 20 / 42, \mathrm{v} / \mathrm{v} / \mathrm{v})$, was flowed through the column at $0.2 \mathrm{~mL} \cdot \mathrm{min}^{-1}$ and the injection volume was set at $5 \mu 1$. MS was operated in positive ion mode with MRM detection using an electrospray voltage of $3000 \mathrm{~V}$, a tube lens offset of respectively $60 \mathrm{~V}$ for $\mathrm{CBZ}$ and $72 \mathrm{~V}$ for epoCBZ and OXC. Capillary and vaporizer temperatures were set respectively at $350{ }^{\circ} \mathrm{C}$ and $300{ }^{\circ} \mathrm{C}$. Nitrogen was used as desolvatation gas and argon as collision gas at a pressure of 1.5 mTorr. Two transitions were monitored for each compound (the first transition for each compound gave the highest signal and was used for quantification) and the collision energy was optimized and indicated in brackets: (i) CBZ: 237> 194 (19V), 237>179 (34V); (ii) epoCBZ: 253> 236 (14V), 253>180 (28V) and (iii) OXC: 253>208 (19V), 253>236 (12V). 
Two MIPs were synthesized using the procedure previously described by our group for the extraction of CBZ from environmental waters [18]. Briefly, the MCBZ was used as template and a ratio 1/4/20 between the template, the monomer and the cross-linker was used. $1 \mathrm{mmol}$ of template and $4 \mathrm{mmol}$ of monomer, respectively TFMAA for MIP 1 and MAA for MIP 2, were mixed with $1.8 \mathrm{~mL}$ of a toluene/DCM $(0.64 / 0.46, \mathrm{v} / \mathrm{v})$ mixture. Then DVB (20 mmol) was added and the mixture was purged during 10 minutes with $\mathrm{N}_{2}$ stream to remove the dissolved oxygen and the initiator. The initiator (AIBN, $0.2 \mathrm{mmol}$ ) was then added into the above mixture and the vial was closed and placed in a water bath at $60^{\circ} \mathrm{C}$ for $48 \mathrm{~h}$. Then, the polymers were crushed, ground automatically in a mixer (MIL MM 301 from Retsch ${ }^{\circledR}$ ) and sieved in a vibratory sieve shaker (Retsch ${ }^{\circledR}$ ). Particles between 25 and $36 \mu \mathrm{m}$ were collected and sedimented with 4 times $5 \mathrm{~mL}$ of $\mathrm{MeOH} /$ water $(80 / 20, \mathrm{v} / \mathrm{v})$ to remove the thin particles and then dried at room temperature. Non-imprinted polymers (NIPs) were synthesized by performing the overall procedure but in the absence of template.

Cartridges of $3 \mathrm{~mL}$ were packed with $55 \mathrm{mg}$ of each MIP or NIP. The polymers were washed with $30 \mathrm{~mL}$ of $\mathrm{MeOH} / \mathrm{AA}(90 / 10, \mathrm{v} / \mathrm{v})$ to remove the template molecule. The washing step was continued until the template could no longer be detected in the washing fraction by LC/MS. Finally, the cartridges were washed with $10 \mathrm{~mL}$ of $\mathrm{MeOH}$ to remove residual AA. The same washing procedure was applied to NIPs.

\section{Repeatability of the extraction procedure in aqueous media}

The retention capacity of the MIPs was first checked in conditions closed to those previously described [18] that ensured a good selectivity for the extraction of CBZ from both MIPs. For the extraction procedure of CBZ, epoCBZ and OXC from pure water, all the cartridges were conditioned with $2.5 \mathrm{~mL}$ of $\mathrm{MeOH}, 1.5 \mathrm{~mL}$ of $\mathrm{HCl}$ $0.1 \mathrm{M}$ and then with $2.5 \mathrm{~mL}$ of water before percolation of $1 \mathrm{~mL}$ of pure water spiked at $5 \mu \mathrm{g} . \mathrm{L}^{-1}$ with $\mathrm{CBZ}$, epoCBZ and OXC. A washing step with $300 \mu \mathrm{L}$ of $\mathrm{HCl} 0.1 \mathrm{M}$ followed by the same volume of water was performed prior to the drying of the cartridge during 20 minutes under vacuum. Then a second washing step with $1 \mathrm{~mL}$ of a mixture of DCM/hexane (40/60, v/v) for MIP/NIP 2 was carried out and finally the analytes were eluted with $1 \mathrm{~mL}$ of $\mathrm{MeOH}$. The second washing and elution fractions were evaporated under $\mathrm{N}_{2}$ stream and resuspended in $100 \mu \mathrm{l}$ of $\mathrm{MeOH} / \mathrm{ACN} / \mathrm{H}_{2} \mathrm{O}(38 / 20 / 42$, v/v/v) before injection in LC/MS-MS.

\section{Extraction procedure applied to urine samples}

150 Before applying urine samples on the MIP/NIP, samples were filtered using $0.2 \mu \mathrm{m}$ syringe filter (Millipore ${ }^{\circledR}$ filters,Merck, Ireland) and the urine samples were further spiked at $20 \mathrm{ng} \cdot \mathrm{L}^{-1}$ and was then diluted 5 times with pure water before percolation. The diluted urine samples were percolated through a conventional sorbent, an Oasis HLB polymer (3cc, $60 \mathrm{mg}$, Waters) and on the MIP 2/NIP 2. The procedure applied to the MIP2/NIP2 was the same exactly as the one previously described for spiked pure water. For Oasis HLB sorbent, the extraction procedure was adapted from [19]. Briefly the cartridge was conditioned with $2 \mathrm{~mL}$ of $\mathrm{MeOH}$ and $2 \mathrm{~mL}$ of water. After this conditioning step, $1 \mathrm{~mL}$ of the diluted urine samples spiked were percolated through the cartridge followed by $2 \mathrm{~mL}$ of water/MeOH (90/10, v/v) mixture as washing step. The sorbent was dried for 20 min under vacuum before the elution of the target analytes with $1 \mathrm{~mL}$ of $\mathrm{MeOH}$. The elution fraction issued of the both $(38 / 20 / 42, v / v / v)$ to be analyzed in LC/MS-MS. 
The elution fraction was injected twice, the first one in MRM mode to quantify CBZ, OXC and epoCBZ in the fraction and to determine the recovery yields, and the second in scan mode $(\mathrm{m} / \mathrm{z}=100-1100)$ in order to visualize the clean-up effect of the extraction on MIP while comparing with results obtained using Oasis HLB.

\section{Evaluation of the matrix effects during LC/MS-MS analysis}

Matrix effects were evaluated by comparing the slopes of the calibration curves constructed in pure water, in the elution fraction resulting from the MIP 2 or from the Oasis HLB. For this, a blank urine sample was diluted five times with water and $1 \mathrm{~mL}$ was percolated through the Oasis HLB cartridge or through the MIP 2. The resulting elution fraction for each support ( $1 \mathrm{~mL}$ for both supports) was equally divided into 4 parts and it was evaporated to dryness under $\mathrm{N}_{2}$ stream. The residue was reconstituted in $20 \mu \mathrm{L}$ of mobile phase $\left(\mathrm{MeOH} / \mathrm{ACN} / \mathrm{H}_{2} \mathrm{O}(38 / 20 / 42\right.$, $\mathrm{v} / \mathrm{v} / \mathrm{v}$ ) containing respectively $0.1,0.2,0.5$ and $2 \mathrm{pg}$ of $\mathrm{CBZ}$, epoCBZ and OXC. Each calibration point was analyzed in MRM mode for performing the quantification of CBZ, OXC and EPOCBZ.

\section{Results}

\section{Development of LC/MS-MS method}

To ensure a sensitive detection of CBZ and two of its analogs epoCBZ and OXC (see Table 1 for structures and $\log \mathrm{P}$ values), in biological samples, it was necessary to optimize first the MS detection parameters (MRM mode) in order to have a highly sensitive and specific method. The optimization was performed for each compound by the direct infusion of a standard solution in MS and led to the choice of two transitions per compound; the most intense ion was used for quantification and the second one for confirmation. Taking into account the hydrophobicity of the analytes and as already reported for real water analysis [18], the LC separation was performed on Varian C18 Omnispher column in isocratic mode. The limit of detection (LOD) and of quantification (LOQ) defined as the concentration value giving rise to a signal-to-noise ratio $(\mathrm{S} / \mathrm{N})$ of 3 and 10 respectively were calculated thanks to the MRM chromatogram obtained for the lowest concentration injected and that gave a S/N ratio higher or equal to 10 (concentration level of $5 \mathrm{ng} . \mathrm{L}^{-1}$ ). The LOD and LOQ values reported in Table 2 were ranging from 3 to $5 \mathrm{ng} . \mathrm{L}^{-1}$ for the three analytes. The calibration curves were linear in the range of concentration level ranging from LOQ to $1 \mathrm{mg} . \mathrm{L}^{-1}$ (see Table 2).

Table 1: Structure and physico-chemical properties of target compounds

\begin{tabular}{|c|c|c|}
\hline Compounds & Structure & Log P \\
\hline Carbamazepine (CBZ) & & 2.77 \\
\hline $\begin{array}{c}\text { Carbamazepine Epoxy 10,11-epoxide } \\
\text { (epoCBZ) }\end{array}$ & & 1.97 \\
\hline
\end{tabular}


191

Table 2 : Equation of calibration curves, LOD and LOQ in pure medium during LC/MS-MS analysis in MRM mode for CBZ, epoCBZ and OXC

\begin{tabular}{|l|c|c|c|c|}
\hline & Quantitation transition & Calibration curve & $\begin{array}{c}\text { LOD } \\
\left(\mathrm{ng} . \mathrm{L}^{-1}\right)\end{array}$ & $\begin{array}{c}\text { LOQ } \\
\left(\mathrm{ng} . \mathrm{L}^{-1}\right)\end{array}$ \\
\hline CBZ & $237>194$ & $\mathrm{y}=987566 \mathrm{x}, \mathrm{r} 2=0.9983$ & 1 & 3 \\
\hline epoCBZ & $253>236$ & $\mathrm{y}=18456 \mathrm{x}, \mathrm{r} 2=0.9935$ & 2 & 5 \\
\hline OXC & $253>208$ & $\mathrm{y}=1567 \mathrm{x}, \mathrm{r} 2=0.9995$ & 2 & 5 \\
\hline
\end{tabular}

\section{Repeatability of the MIP synthesis and of the extraction procedure in pure water}

In a previous study from our group, several conditions of synthesis of MIPs were screened by varying the nature of the monomers, of the cross-linker and of the porogenic solvent. Two MIPs have allowed the selective extraction of CBZ from pure organic media with similar performances. These two MIPs, synthesized using TFMAA or MAA as monomer and DVB as cross-linker in a toluene/dichloromethane mixture, were then studied directly in more detail in real water by optimizing, for each of them, the washing step that should ensure a high retention of CBZ on MIP and a lower one on NIP after the percolation of $25 \mathrm{~mL}$ of spiked tap water. The MIP produced with MAA appeared as slightly more selective towards CBZ that the one produced with TFMAA. Indeed, the comparison of the selectivity of these MIPs, achieved by comparing the ratios between the recoveries obtained on MIP and on NIP for a given molecule, were 3.07 for CBZ for TFMAA-based MIP and 4.41 for the MAA-based MIP. Therefore, this later was applied to the extraction of CBZ, OXC and epoCBZ from mineral and surface water without a real optimization of the extraction conditions for these two compounds. Nevertheless, high recoveries were obtained for the three compounds in real environmental water sample (with recoveries between 60 and $69 \%$ on the MIP and of only between 19 and $27 \%$ on the NIP) [18]. Here, the purpose was slightly different. Indeed the extraction procedure had to be optimized in order to allow not only the selective extraction of CBZ but also the extraction of two of its analogs, epoCBZ and $\mathrm{OXC}$ from urine that is a more complex matrix than mineral and surface water previously studied and available in smaller volume than environmental waters. These new objectives could imply that the MIP previously identified as the best one for the selective trapping of CBZ in environmental samples could not be the best one for the trapping of CBZ and of its analogs from urine samples. Then, the two most promising MIPs previously synthesized using the MCBZ as template, TFMAA or MAA as monomer (named respectively MIP 1 and MIP 2) were again synthesized as their corresponding NIPs. To assess their selectivity, the retention in pure water of the three target compounds was first studied by applying a procedure of extraction very closed to the one previously applied to surface water but to a reduced volume of water ( $1 \mathrm{~mL}$ instead of $25 \mathrm{~mL}$ ) and by optimizing again the washing conditions (different DCM/hexane ratios were assayed) to ensure a high selectivity for the three targets. The highest recoveries on the MIPs while maintaining low recoveries on the NIPs 
were obtained using a DCM/hexane mixture of 70/30 (v/v), instead of 80/20 previously fixed, for MIP 1 and of $60 / 40$, as previously, for MIP 2. As shown on Figure 1, after percolation of pure water spiked with CBZ, epoCBZ and OXC at $5 \mu \mathrm{g} \cdot \mathrm{mL}^{-1}$, both MIPs provided (i) a high retention not only for CBZ but also for epoCBZ and OXC with high extraction recoveries in the elution fraction between 79 and $82 \%$ for the three compounds and (ii) a high selectivity illustrated by extraction recoveries below $20 \%$ on both corresponding NIPs. Figure 1 also illustrates the contribution of the washing solution on the removal of non-specific interactions, more than $80 \%$ of the target analytes were lost from the NIP during this step. The high extraction recoveries in the elution fraction and the selectivity for CBZ observed on these two couple of supports are in good agreement with those described previously for CBZ extracted from aqueous media [18] thus indicating also the reliability of the synthesis of these MIP/NIPs even if the conditions of evaluation were not exactly the same. It can also be noticed a high repeatability of the extraction procedure on both MIPs with RSD values lower than $8 \%$ for recoveries on MIPs ( $n=3)$. The recoveries obtained using the two couples of MIP/NIPs for CBZ, epoCBZ and OXC, are very similar thus rendering difficult their selection. Nevertheless, MIP 2 (synthesized with MAA as monomer) have previously shown the highest selectivity in real environmental samples towards CBZ [18] and a slightly higher recovery for epoCBZ was observed associated with a lower recovery on the NIP 2 than on the NIP 1 during this study. Then MIP 2 was selected for the rest of the study.



CBZ

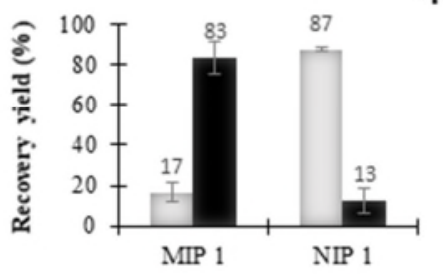

epoCBZ


OXC
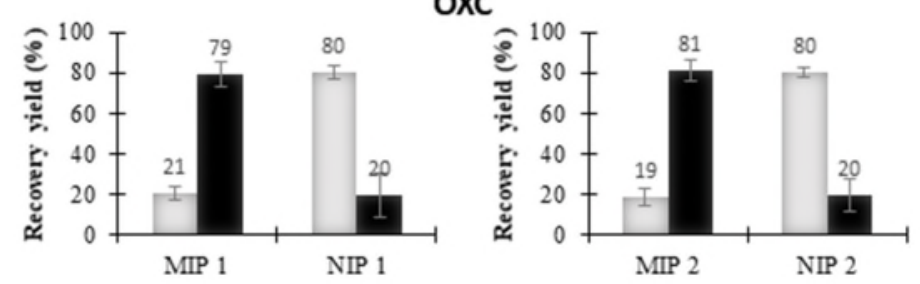

Figure 1: Extraction profiles obtained when percolating pure water spiked at $5 \mu \mathrm{g} . \mathrm{L}^{-1}$ with CBZ, epoCBZ and OXC on the both synthesized MIPs and NIPs. Extraction procedure: percolation of $1 \mathrm{~mL}$ spiked pure water, washing with $1.5 \mathrm{~mL}$ of a mixture DCM/Hexane, 70/30 (v/v) for MIP/NIP 1 and 60/40 (v/v) for MIP/NIP 2; elution with $1 \mathrm{~mL} \mathrm{MeOH}$. The grey and black bars correspond to the recoveries in the washing and elution fractions respectively. 
After these promising results obtained for the selective extraction of the three target analytes from spiked pure water, the performance of the MIP 2 was evaluated for the extraction of the target molecules from urine samples. The optimized extraction procedure was first applied to a non-spiked urine sample and no target analytes were detected in the eluate. The extraction procedure was then applied to this urine sample spiked at $20 \mathrm{ng} . \mathrm{L}^{-1}$ with each compounds. The extraction recoveries in the elution fraction corresponding to the MIP and the NIP are reported in the Table 3. The recoveries on the elution fraction of the MIP were higher than $82 \%$ for the three compounds and lower than $20 \%$ for the NIP thus highlighting the selectivity of the extraction procedure on MIP applied to a urine sample. The RSD values $(n=3)$ describing the repeatability of the extraction procedure applied to urine samples were acceptable for this concentration level of $20 \mathrm{ng} \cdot \mathrm{L}^{-1}$ and in the range of $13 \%$ to $23 \%$.

Table 3: Extraction recoveries ( $\% \pm \mathrm{RSD}$ values, $\mathrm{n}=3$ ) of CBZ, epoCBZ and OXC from urine on the MIP/NIP 2 and on Oasis HLB. Extraction procedure: percolation of $1 \mathrm{~mL}$ of diluted urine sample (urine spiked at 20 ng. $\mathrm{L}^{-1}$ and diluted by a factor 5 with pure water); washing with $1.5 \mathrm{~mL}$ of a mixture DCM/hexane, $60 / 40(\mathrm{~V} / \mathrm{V})$ for MIP/NIP2 or with $1.5 \mathrm{~mL}$ of $\mathrm{H}_{2} \mathrm{O} / \mathrm{MeOH}, 90 / 10$, v/v for Oasis HLB; elution with $1 \mathrm{~mL} \mathrm{MeOH}$. ND : Not Detected

\begin{tabular}{|l|c|c|c|}
\hline & MIP 2 & NIP 2 & Oasis \\
\hline CBZ & $82 \pm 23$ & $20 \pm 7$ & $74 \pm 11$ \\
\hline epoCBZ & $87 \pm 17$ & ND & $88 \pm 30$ \\
\hline OXC & $106 \pm 13$ & $4 \pm 10$ & $31 \pm 8$ \\
\hline
\end{tabular}

In order to highlight the potential of the MIP 2, its performances in term of recovery yield and of cleaning capacity were compared to those obtained with a conventional Oasis HLB sorbent commonly applied to the extraction of drugs from biological samples. For this purpose, the same urine sample spiked at $20 \mathrm{ng} . \mathrm{L}^{-1}$ was percolated on Oasis HLB cartridge following a procedure previously reported [19]. The extraction recovery obtained using Oasis HLB for CBZ, epoCBZ and OXC are also reported in Table 3. Recoveries obtained using Oasis HLB were similar for $\mathrm{CBZ}$ and epoCBZ as those obtained on MIP 2 with recoveries of 74 and $88 \%$ respectively using Oasis HLB and 82 and $87 \%$ respectively using MIP 2. In return, a recovery of only $31 \% \pm 8 \%$ was obtained using the Oasis HLB instead of $106 \pm 13 \%$ on MIP 2 for OXC. This compound being less polar than epoCBZ (see log P value, Table 1), it must be better retained than epoCBZ in this reverse phase mode retention process. Therefore, a matrix effect during the LC/MS quantification of this compound was suspected. The comparison of the LC/MS chromatogram in scan mode, and in MRM mode resulting from the use of the MIP (Figure 2A) and the use of Oasis HLB sorbent (Figure 2B) for epoCBZ and OXC clearly illustrates that the elution fraction resulting from the Oasis HLB contained more interfering compounds than the elution fraction of the MIP: (i) higher baseline signal and presence of many peaks in scan mode and (ii) an important noise in MRM mode particularly visible for the transition of the OXC (Figure 2B). The cleaner extract obtained using the MIP (Figure 2A) should provide more repeatable quantitation as demonstrated by the lower RSD values observed for the quantification in the elution fraction of the target analytes of the MIP as compared as those on Oasis HLB that reached 30\% for epoCBZ (Table 3). The S/N ratio observed in MRM mode for CBZ, OXC and epoCBZ (191, 33 and 54 respectively) highlighted the ability of the MIP 2 coupled with LC/MS-MS analysis to quantify the CBZ, OXC and epoCBZ at a 

applied to the MIP.
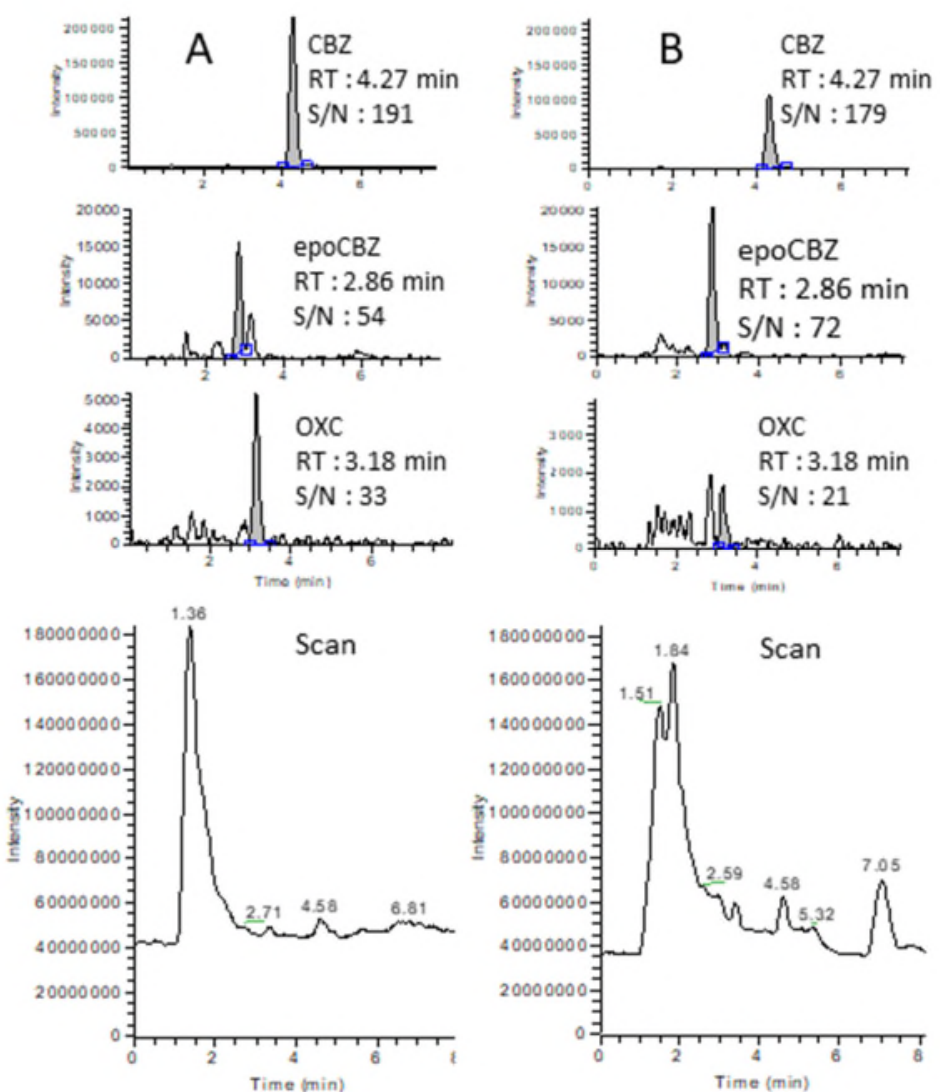

Figure 2 : LC/MS analysis in scan mode and in MRM mode of the elution fraction obtained after the percolation of urine spiked at $20 \mathrm{ng} . \mathrm{L}^{-1}$ on MIP 2 (A) and on Oasis HLB (B). From the top to the bottom: MRM chromatograms (CBZ : 237> 194, epoCBZ: 253 $\rightarrow 236$ and OXC:253>208), and LC/MS chromatograms in scan mode (m/z = 1001100).

\section{Evaluation of the matrix effects during LC/MS-MS analysis}

Potential matrix effects were evaluated more in detail for epoCBZ and OXC by comparing the slopes of three different calibration curves. The first calibration curve, used as reference, was constructed by injecting solutions corresponding to the composition of the mobile phase spiked with epoCBZ and OXC. The second and third one were constructed by injecting in LC/MS-MS the elution fraction of a non-spiked and spiked (with different amounts of the target molecules) new urine sample, after its percolation on Oasis HLB or on the MIP. The resulting calibration curves are reported in Figure 3. The slope of the calibration curves for epoCBZ and OXC after applying the urine sample on MIP are very close to those obtained for spiked pure media, thus indicating that the selectivity provided by MIP makes it an effective tool for the matrix removal. On the other hand, the large difference between the slope obtained for spiked pure water and spiked extract of urine passed through Oasis HLB highlights a strong matrix effect when analyzing this new urine sample that could give rise to an overestimation of the amount of those two compounds in urine. Indeed, the signal was exhausted for both compounds (around 56\% and 23\% for epoCBZ and OXC respectively). This problem of quantification was easily circumvented by using the MIP. 

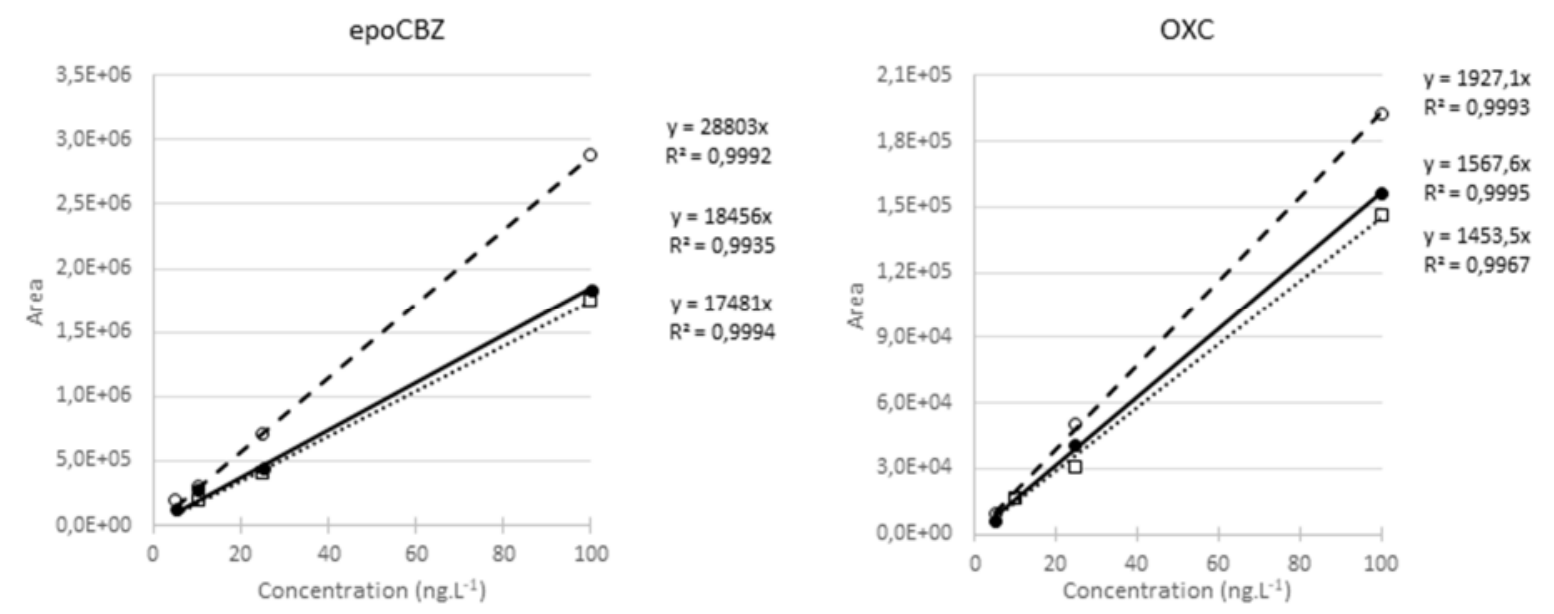

Figure 3: Calibration curves for epoCBZ and OXC obtained for spiked pure water (plain line), spiked extract of urine sample obtained after SPE on MIP (dotted line) or on Oasis HLB (dash line).

\section{Conclusion}

After a first study focused on the screening of the synthesis conditions of the MIP/NIP capable of selectively extract CBZ from environmental samples [18], the two most promising MIPs were assayed for their ability to selectively extract the two drugs $\mathrm{CBZ}$ and $\mathrm{OXC}$ and the metabolite epoCBZ from human urine. A high retention and a high selectivity for the three structural analogs were demonstrated for a concentration level of only few ng. L $^{-}$ ${ }^{1}$ in urine. The sample treatment with the MIP also demonstrated a more efficient clean-up of the human urine compared with a conventional Oasis HLB sorbent. It therefore appeared crucial to readjust (re-optimize) the extraction procedure applied to the MIP for each matrix. However, an application for the quantification of the three targeted compounds from other biological fluids such as plasma or saliva (noninvasive sampling), which is now considering like an alternative for the determination of drug intake [20], could be considered in the future.

\section{Acknowledgments}

This work was supported by the French National Research Agency (ANR Program: ANR-15-CE04-0012, project MIP_WQT).

\section{Compliance with Ethical Standards}

Conflicts of interest: Authors declare that they have no conflict of interest

Research involving Human Participants and/or Animals: Informed consent was obtained from all individual participants included in this study.

\section{References}

1. Brezina E, Prasse C, Meyer J, et al (2017) Investigation and risk evaluation of the occurrence of carbamazepine, oxcarbazepine, their human metabolites and transformation products in the urban water cycle. Environmental Pollution 225:261-269. https://doi.org/10.1016/j.envpol.2016.10.106 
2. Datar PA (2015) Quantitative bioanalytical and analytical method development of dibenzazepine derivative, carbamazepine: A review. Journal of Pharmaceutical Analysis 5:213-222. https://doi.org/10.1016/j.jpha.2015.02.005

3. Fedorova G, Ben Ari J, Tadmor G, et al (2016) Environmental exposure to pharmaceuticals: A new technique for trace analysis of carbamazepine and its metabolites in human urine. Environmental Pollution 213:308313. https://doi.org/10.1016/j.envpol.2016.02.027

4. Chen Szu-Ying, Chen Wen-Chi, Chang Sarah Y. (2018) Cyclodextrin-assisted dispersive liquid-liquid microextraction for the preconcentration of carbamazepine and clobazam with subsequent sweeping micellar electrokinetic chromatography. Journal of Separation Science 41:1871-1879. https://doi.org/10.1002/jssc.201701096

5. Zhang R, Wang S, Yang Y, et al (2018) Modification of polydopamine-coated Fe3O4 nanoparticles with multi-walled carbon nanotubes for magnetic-mu-dispersive solid-phase extraction of antiepileptic drugs in biological matrices. Analytical and bioanalytical chemistry 410:3779-3788. https://doi.org/10.1007/s00216018-1047-1

6. Behbahani M, Najafi F, Bagheri S, et al (2013) Application of surfactant assisted dispersive liquid-liquid microextraction as an efficient sample treatment technique for preconcentration and trace detection of zonisamide and carbamazepine in urine and plasma samples. Journal of Chromatography A 1308:25-31. https://doi.org/10.1016/j.chroma.2013.07.088

7. Beltran A, Caro E, Marce RM, et al (2007) Synthesis and application of a carbamazepine-imprinted polymer for solid-phase extraction from urine and wastewater. Analytica Chimica Acta 597:6-11

8. Beltran A, Marcé RM, Cormack PAG, Borrull F (2009) Synthesis by precipitation polymerisation of molecularly imprinted polymer microspheres for the selective extraction of carbamazepine and oxcarbazepine from human urine. Journal of Chromatography A 1216:2248-2253

9. Dai C, Geissen S-U, Zhang Y, et al (2010) Performance evaluation and application of molecularly imprinted polymer for separation of carbamazepine in aqueous solution. Journal of Hazardous Materials 184:156-163. https://doi.org/10.1016/j.jhazmat.2010.08.018

10. Esfandyari-Manesh Mehdi, Javanbakht Mehran, Atyabi Fatemeh, et al (2011) Effect of porogenic solvent on the morphology, recognition and release properties of carbamazepine-molecularly imprinted polymer nanospheres. Journal of Applied Polymer Science 121:1118-1126. https://doi.org/10.1002/app.33812

11. Esfandyari-Manesh Mehdi, Javanbakht Mehran, Atyabi Fatemeh, Dinarvand Rassoul (2012) Synthesis and evaluation of uniformly sized carbamazepine-imprinted microspheres and nanospheres prepared with different mole ratios of methacrylic acid to methyl methacrylate for analytical and biomedical applications. Journal of Applied Polymer Science 125:1804-1813. https://doi.org/10.1002/app.36288

12. Esfandyari-Manesh M, Javanbakht M, Dinarvand R, Atyabi F (2012) Molecularly imprinted nanoparticles prepared by miniemulsion polymerization as selective receptors and new carriers for the sustained release of carbamazepine. J Mater Sci: Mater Med 23:963-972. https://doi.org/10.1007/s10856-012-4565-y

13. Dai C, Zhang J, Zhang Y, et al (2013) Removal of carbamazepine and clofibric acid from water using double templates-molecularly imprinted polymers. Environ Sci Pollut Res 20:5492-5501. https://doi.org/10.1007/s11356-013-1565-5

14. Zhang Y-L, Zhang J, Dai C-M, et al (2013) Sorption of carbamazepine from water by magnetic molecularly imprinted polymers based on chitosan-Fe3O4. Carbohydrate Polymers 97:809-816. https://doi.org/10.1016/j.carbpol.2013.05.072

15. Lee J-C, Kim C-R, Byun H-S (2014) Synthesis and adsorption properties of carbamazepine imprinted polymer by dispersion polymerization in supercritical carbon dioxide. Korean Journal of Chemical Engineering 31:2266-2273. https://doi.org/10.1007/s11814-014-0178-0 
16. Khalilian Faezeh, Ahmadian Setareh (2016) Molecularly imprinted polymer on a SiO2-coated graphene oxide surface for the fast and selective dispersive solid-phase extraction of Carbamazepine from biological samples. Journal of Separation Science 39:1500-1508. https://doi.org/10.1002/jssc.201501392

17. Asgari S, Bagheri H, Es-haghi A, AminiTabrizi R (2017) An imprinted interpenetrating polymer network for microextraction in packed syringe of carbamazepine. Journal of Chromatography A 1491:1-8. https://doi.org/10.1016/j.chroma.2017.02.033

18. Khadirvel P, Combès A, Bordron L, Pichon V (2018) Rational designing and development of water compatible molecularly imprinted polymers for the selective extraction of carbamazepine from environmental waters. Submitted:

19. Fortuna A, Sousa J, Alves G, et al (2010) Development and validation of an HPLC-UV method for the simultaneous quantification of carbamazepine, oxcarbazepine, eslicarbazepine acetate and their main metabolites in human plasma. Anal Bioanal Chem 397:1605-1615. https://doi.org/10.1007/s00216-0103673-0

20. Neumann J, Beck O, Dahmen N, Böttcher M (2018) Potential of Oral Fluid as a Clinical Specimen for Compliance Monitoring of Psychopharmacotherapy. Therapeutic Drug Monitoring 40:245. https://doi.org/10.1097/FTD.0000000000000493 